\title{
Nutritional biomarkers to assess compliance to dietary advice given in an integrated dietary intervention: results from the CRESSIDA Study
}

\author{
J. Darzi ${ }^{1}$, D. P. Reidlinger ${ }^{1}$, W. L. Hall ${ }^{1}$, Z. Maniou $^{1}$, V. Govoni ${ }^{1}$, D. R. Taylor $^{2}$, P. Seed ${ }^{3}$, \\ P. J. Chowienczyk ${ }^{4}$ and T. A. B. Sanders ${ }^{1}$ \\ ${ }^{1}$ Diabetes and Nutritional Sciences Division, King's College London, London SE1 9NH, UK, ${ }^{2}$ Department of Clinical \\ Biochemistry, King's College Hospital NHS Foundation Trust, London SE5 9RS, UK, ${ }^{3}$ Division of Women's Health and \\ ${ }^{4}$ Cardiovascular Division, King's College London, St Thomas' Hospital, London SE1 7EH, UK
}

The CRESSIDA Study (Cardiovascular risk Reduction Study Supported by an Integrated Dietary Approach) was designed to systematically evaluate the Department of Health guidelines for the prevention of cardiovascular disease (CVD). The study aimed to investigate effects of consuming a cardioprotective diet for 12 weeks on CVD risk factors in comparison to a traditional UK (control) diet in a parallel RCT (ISRCTN92382106).

One hundred and sixty five healthy non-smoking volunteers were randomised to treatment and 162 completed the intervention. The cardioprotective diet aimed to supply 1-2 portions of oily fish per week, five portions of fruit and vegetables per day, wholegrain cereals intake to account for $>50 \%$ cereal intake and to restrict saturated fatty acid intake to $<10 \%$ energy, salt intake to $<6 \mathrm{~g}$ per day and restrict intake of added sugars. Participants were given individualised dietary advice with regular follow up by a dietitian throughout the study. Compliance to dietary advice was assessed by nutritional biomarkers, including erythrocyte lipids, plasma alkylresorcinols (AR) and urinary sucrose and sodium excretion to assess intake of oily fish, whole grains ${ }^{(1)}$, added sugars ${ }^{(2)}$ and salt respectively. Completeness of urine collection was ascertained using the para-amino benzoic acid (PABA) recovery method.

Calculated nutrient intakes from 4-day food diaries suggested the participants complied with dietary advice and this was corroborated by the changes in nutritional biomarkers shown in the table below. A salt intake of less than $6 \mathrm{~g}$ per day corresponds to a urine sodium excretion of less than $100 \mathrm{mmol}$ per day. We found the HPLC/MS/MS method we developed was sensitive to decreases in added sugars to the diet. These findings illustrate the utility of biomarkers in monitoring compliance to complex dietary interventions.

\begin{tabular}{|c|c|c|c|c|c|}
\hline & & \multicolumn{2}{|c|}{ Cardioprotective $n 80$} & \multicolumn{2}{|c|}{ Control $n 82$} \\
\hline & & Mean & $95 \% \mathrm{CI}$ & Mean & $95 \% \mathrm{CI}$ \\
\hline \multirow[t]{2}{*}{ Urinary $\mathrm{Na}(\mathrm{mmol} / 24 \mathrm{~h})^{\dagger \S}$} & Baseline & 123.3 & $(114,132)$ & 142.7 & $(130,156)$ \\
\hline & Follow up & $93.0 *$ & $(84,102)$ & 142.1 & $(130,154)$ \\
\hline \multirow[t]{2}{*}{ Urinary sucrose $(\mu \mathrm{mol} / 24 \mathrm{~h})^{\dagger \S}$} & Baseline & 41.3 & $(30,56)$ & 44.1 & $(34,56)$ \\
\hline & Follow up & $28.5^{*}$ & $(22,38)$ & 61.6 & $(49,77)$ \\
\hline \multirow[t]{2}{*}{ Plasma AR $(\mathrm{nmol} / \mathrm{L})^{\dagger}$} & Baseline & 68.8 & $(58,81)$ & 70.1 & $(58,85)$ \\
\hline & Follow up & $84.6^{*}$ & $(73,98)$ & 57.1 & $(47,68)$ \\
\hline \multirow[t]{2}{*}{ Erythrocyte DHA (wt\%) } & Baseline & 5.51 & $(5.10,5.92)$ & 5.87 & $(5.52,6.22)$ \\
\hline & Follow up & $6.37 *$ & $(5.95,6.79)$ & 5.38 & $(5.03,5.73)$ \\
\hline
\end{tabular}

* Significant treatment effect $P<0.001$; values are mean or †geometric mean, $\S$ corrected for PABA recovery.

This work was supported by the Food Standards Agency and the Department of Health.

1. Landberg RE, Aman P, Hallmans G et al. (2013) Eur J Clin Nutr 67, 259-263.

2. Joosen AM, Kuhnle GC, Runswick SA et al. (2008) Int J Obes 32, 1736-1740. 